\title{
High-frequency Turnover in Pulsar Spectra
}

\author{
J. Kijak \\ Institute of Astronomy, University of Zielona Góra, Poland \\ O. Maron \\ Institute of Astronomy, University of Zielona Góra, Poland \\ Max-Planck-Institut für Radioastronomie, Bonn, Germany
}

\begin{abstract}
Spectra of several pulsars with turnover at unusually high frequencies $\sim 1 \mathrm{GHz}$ are presented. These are relatively young pulsars with DM typically larger than for pulsars showing a low-frequency turnover.
\end{abstract}

\section{Introduction}

In general, pulsar spectra should represent intrinsic characteristics of pulsars and tell us about the emission mechanism. A typical pulsar spectrum is steep compared with spectra of other radio objects and can be described by a simple power law. However, some pulsars show an unusual spectral behavior: the lowfrequency turnover $(\sim 100 \mathrm{MHz})$ and the high-frequency break $(>1 \mathrm{GHz})$ in the power law spectrum (Malofeev et al. 1994; Maron et al. 2000). It is still an open question whether the cause of the turnover is some kind of absorption in the magnetosphere, loss of efficiency of the emission mechanism (Sieber 1973) or an interstellar effect (Sieber 2002). Here we present several pulsars with a turnover at unusually high frequencies $\sim 1 \mathrm{GHz}$ (type $\mathrm{T}_{\mathrm{H}}$ ).

\section{Turnover at High Frequency}

In Maron et al. (2000) we presented a large number of pulsar spectra in a wide frequency range and found two young pulsars (B1823-13 and B1838-04) that indicate turnover at $\sim 1 \mathrm{GHz}$. The collected pulsar spectra were analyzed again adding new flux measurements at high frequencies $(>1.4 \mathrm{GHz})$ made with the Effelsberg radio telescope and published in recent papers. As a result, another four pulsars of type $T_{H}$ were found (see Fig. 1). In addition, five pulsars were selected as $\mathrm{T}_{\mathrm{H}}$ candidates because their spectra indicate a possible turnover. Several objects show cut-off in spectra around $1 \mathrm{GHz}$ (meaning, no detection at low frequencies) as a result of a high value of dispersion measure DM. However, one cannot exclude existence of $T_{H}$ in these pulsars, so we treat them as candidates for $\mathrm{T}_{\mathrm{H}}$ as well (see Table 1). 


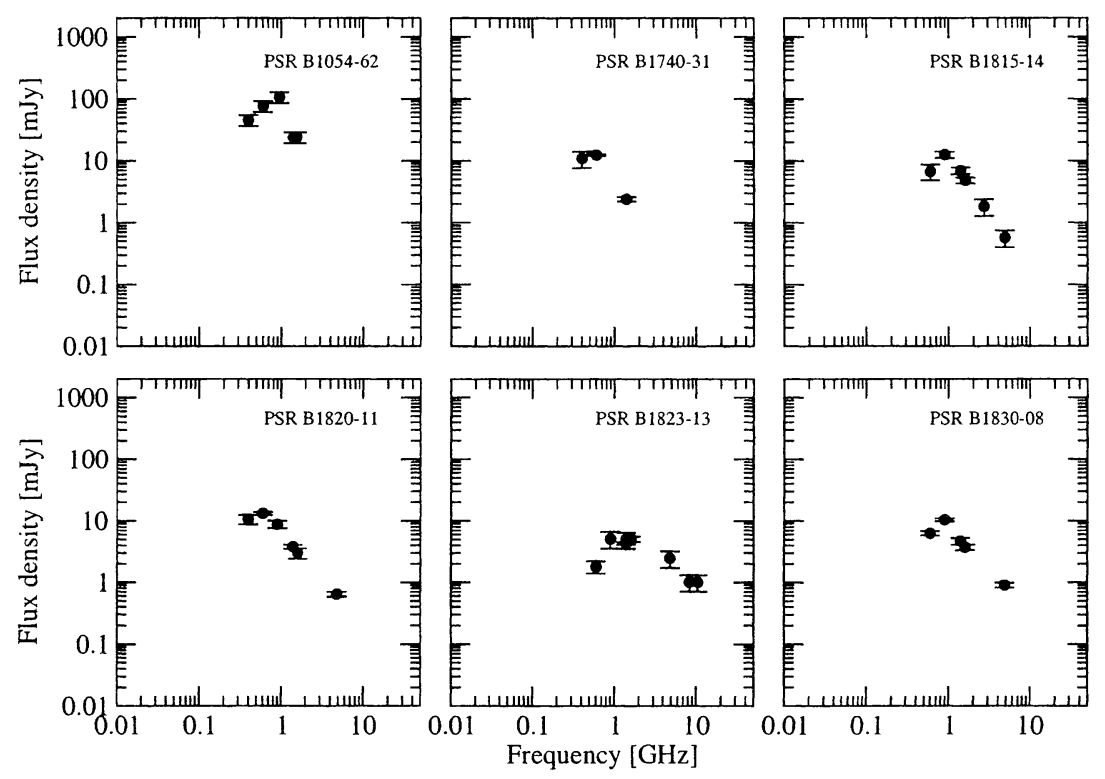

Figure 1. Pulsars with high-frequency turnover.

Pulsars with high-frequency turnover are relatively young compared to others with a low-frequency turnover and they also have large values of DM.

Table 1. List of candidates for turnover at high frequency. Objects with cut-off in spectra are marked with stars.

\begin{tabular}{|c|c|c|c|c|}
\hline $\bar{B} 1240-64$ & $\overline{\mathrm{B} 1557-50^{\star}}$ & B1641-45 & $\mathrm{B} 1714-34^{\star}$ & $\overline{\mathrm{B} 1736-31^{\star}}$ \\
\hline B1740-31 & B1750-24 & B1758-23* & $\mathrm{B} 1820-14^{\star}$ & B1822-14* \\
\hline B1823-11 & $\mathrm{B} 1832-06^{\star}$ & $\mathrm{B} 1849+00^{\star}$ & & \\
\hline
\end{tabular}

Acknowledgments. This work was supported in part by the Polish State Committee for Scientific Research Grant 2 P03D 008 19. JK acknowledges partial support from the IAU.

\section{References}

Malofeev, V. M., Gil, J. A., Jessner, A., Malov, I. F., Seiradakis, J. H., Sieber, W., \& Wielebinski, R. 1994, A\&A, 285, 201

Maron, O., Kijak, J., Kramer, M., \& Wielebinski, R. 2000, A\&AS, 147, 195

Sieber, W. 1973, A\&A, 28, 237

- 2002, in MPE Rep. 278, Neutron Stars, Pulsars and Supernova Remnants, eds. W. Becker, H. Lesch, \& J. Trümper, (Garching: MPE), p. 171 